\title{
A Survey of U.S. and Canadian Hospitals' Paediatric Massive Transfusion Protocol Policies
}

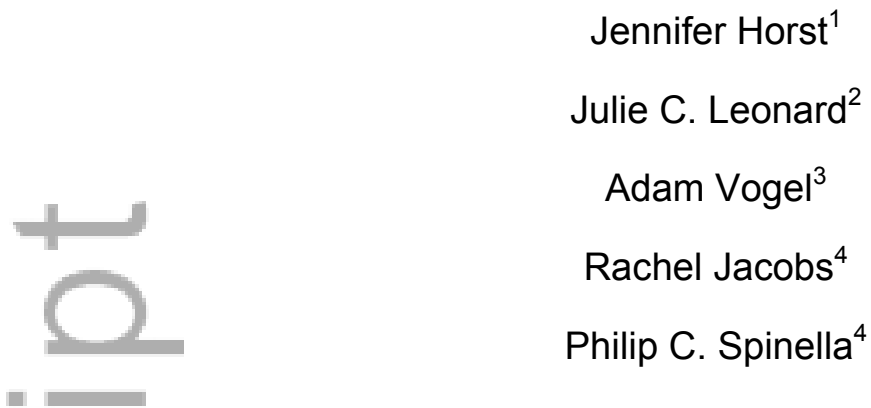

1. Division of Emergency Medicine, Department of Paediatrics, Washington University in St. Louis, St Louis, MO

2. Section of Emergency Medicine, Department of Paediatrics, Nationwide Children's Hospital and the Ohio State University, Columbus, Ohio.

3. Division of Paediatric Surgery, Department of Surgery, Washington University in St. Louis, St. Louis, MO

4. Division of Critical Care, Department of Paediatrics, Washington University in St. Louis, St. Louis, MO

\section{Address Correspondence:}

Jennifer Horst

Campus Box $8116,9^{\text {th }}$ Floor NWT

1 Children's Place

St Louis, MO 63110

Phone: 314-747-3350

Email: horst_j@kids.wustl.edu

Conflicts of Interest: None to Disclose

Funding: None

Word Count: 4574

Running title: Survey of U.S. and Canadian Hospitals' Paediatric Massive Transfusion Protocol Policies

Acknowledgements: All authors contributed to the development, writing and editing of this manuscript.

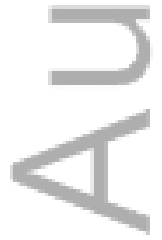

This is the author manuscript accepted for publication and has undergone full peer review but has not been through the copyediting, typesetting, pagination and proofreading process, which may lead to differences between this version and the Version of Record. Please cite this article as doi: $10.1111 /$ tme.12277 


\section{Abstract}

Background: Trauma is the leading cause of death in children $>1$ year of age, with haemorrhage as the most common cause of medically preventable deaths. While massive transfusion protocols (MTPs) have been investigated and used in adults to reduce death from haemorrhage, there are a paucity of published data on MTP practices and outcomes in children. This study aimed to survey current MTP policies and the frequency of activation at paediatric care centres.

Study Design and Methods: We conducted a survey of MTPS at hospitals in the U.S. and Canada, including children's general hospitals, children's specialty hospitals, and children's units in general hospitals. We collected information on how the MTP is activated, what therapeutics are given, frequency of its use, and how it is audited for compliance.

Results: Forty-six survey responses were analysed. Physician discretion was the most common activation criteria (89\%). A majority of sites $(78 \%)$ targeted a "high" $(\geq 1: 2)$ ratio of plasma to red blood cells (RBC). Fifteen percent of sites use antifibrinolytics in their MTPs. Eighty nine percent of sites have type-O RBC units and $48 \%$ of sites had thawed plasma units stored in an immediately available location.

Conclusion: There is a wide variation in MTPs among paediatric hospitals with regard to both activation criteria and products administered. This underscores the need for future prospective studies to determine the most effective resuscitation methods for paediatric populations to improve outcomes and therapeutic safety for massive bleeding.
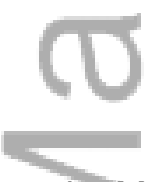

Keywords: Massive Transfusion Protocol, Haemorrhage, Paediatrics, Red Blood Cells, Damage Control Resuscitation

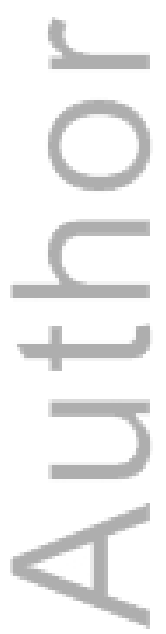




\section{Introduction}

Trauma is the most common cause of death in children $>1$ year of age (Center for Disease Control, 2010). Haemorrhage, the most common cause of medically preventable death for patients with traumatic injuries, typically occurs within 6 hours from the initiation of bleeding(Demetriades, Murray et al., 2004). Additionally, life-threatening bleeding in children may also occur from aetiologies other than trauma, such as intra and post-operative bleeding, gastrointestinal bleeding, and disseminated intravascular coagulation (DIC). In order to prevent death from haemorrhagic shock, transfusion strategies for massive haemorrhage are evolving, based on recent military and adult civilian trauma data (Spinella and Holcomb, 2009). Damage Control Resuscitation (DCR) is the term used to describe the current treatment approach for traumatic haemorrhagic shock (Spinella and Holcomb, 2009). The goal of DCR is to prevent death from haemorrhage by the rapid identification and treatment of traumatic shock and coagulopathy.

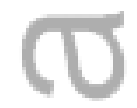

In retrospective studies, DCR principles have been independently associated with reduced mortality in adults with traumatic and intra-operative bleeding (Borgman, Spinella et al., 2007; Gunter, Au et al., 2008; Perkins, Cap et al., 2009; Spinella and Holcomb, 2009; Holcomb, Wade et al., 2011). Massive Transfusion Protocols (MTPs) have been developed to implement some aspects of DCR principles. In adults with traumatic injury, MTPs have been associated with reduced mortality (Cotton, Gunter et al., 2008; Gunter, Au et al., 2008; O'Keeffe, Refaai et al., 2008; Cotton, Au et al., 2009; Dente, Shaz et al., 2009). However, in some institutions, MTPs are applied to all patients with massive bleeding and not just those with traumatic injury. In addition, the current literature on MTPs in children is scant and includes case reports, as well as studies discussing the feasibility of implementing MTPs at children's hospitals, the risk of hyperkalaemia, and the ability to continuously monitor 
haemoglobin during the MTP (Nylund, Borgman et al., 2009; Dressler, Finck et al., 2010; Hendrickson, Shaz et al., 2012; Agrawal, Beethe et al., 2013; Nosanov, Inaba et al., 2013). There is one prospective cohort study of paediatric patients who received blood products according to a MTP versus no MTP. The MTP group consumed a higher overall amount of blood products, but there was no significant difference in the transfused plasma to red blood cell (RBC) ratio (Chidester, Williams et al., 2012). There are no paediatric multi-centre data published on the epidemiology of MTP activations, which DCR therapies are most commonly used in children, and associated outcomes according to the cause of massive bleeding.

Despite a paucity of evidence supporting DCR in children, it is being generalized to paediatric practice within MTPs for both traumatic and non-traumatic aetiologies (Nylund, Borgman et al., 2009; Dressler, Finck et al., 2010; Chidester, Williams et al., 2012; Agrawal, Beethe et al., 2013; Nosanov, Inaba et al., 2013; Palmieri, Greenhalgh et al., 2013). Therefore, it is essential that DCR be studied specifically in children. Anatomical and physiological differences in children may alter the efficacy and safety of certain DCR principles compared to adults. Children have reduced blood volume compared to adults and, as a result, may not tolerate large blood volume losses relative to a similar injury. Conversely, the lack of cardiac and vascular disease in children may also permit for increased ability to compensate during haemorrhagic shock. In addition, haemostasis is known to change through infancy and also in females during their first menstrual cycle (Guzzetta and Miller, 2011; Appel, Grimminck et al., 2012; Jaffray and Young, 2013). It is possible that these developmental changes may have implications for the efficacy and safety of haemostatic therapies in children with life-threatening bleeding (Andrew, Vegh et al., 1992). As a result, the use of haemostatic adjuncts, such as tranexamic acid or prothrombin complex concentrates, may have an improved safety profile in children compared to adults. 
The objective of this survey was to determine current MTP policies and the frequency of MTP activations at paediatric hospitals. We hypothesized that there is a high degree of variability in MTPs in hospitals caring for children. The data from this survey will be used to develop a prospective observational study at paediatric tertiary care centres, which ultimately will allow development of trials to determine which DCR concepts are appropriate to apply to paediatric populations with life-threatening bleeding.

\section{Materials and Methods}

We conducted a survey of MTPs at paediatric hospitals, including children's general hospitals, children's specialty hospitals, and children's units in general hospitals. Study centres were identified through a query of the National Association of Children's Hospitals and Related Institutions (NACHRI) database. The survey was developed in REDCap (Research Electronic Data Capture) with the assistance of an expert in survey development and was pilot tested for clarity of questions at three study centres. An email that introduced the survey and provided instructions was sent electronically to paediatric trauma program directors at each site, with the rationale that, in most circumstances, trauma services require the development of MTPs for certification. The email address for the trauma directors from each institution was obtained via multiple methods, including Internet searches and review of contact lists from multiple research networks. The trauma program directors could delegate completion of the survey to another person in a different specialty at the site if that individual was more familiar with specific aspects of their MTP (i.e. the blood bank director or emergency medicine director). Once centres completed the survey, we removed them from our distribution list and they were not contacted further. 
We solicited survey responses three times via a secured web-based interface to REDCap on January 15, January 27 and February 4th of 2014. Only one response per site was permitted. The last survey was accepted on March 31, 2014. Of note, the survey was administered prior to the publication of the PROPPR (Pragmatic, Randomized Optimal Platelet and Plasma Ratios) trial (Holcomb, Tilley et al., 2015). Survey data were analysed using descriptive statistics and Chi Square or Fischer Exact tests as appropriate (IBM SPSS Statistics for Macintosh, Version 22.0.0.0). This study underwent administrative review by the institutional review board at Washington University in St. Louis and it was deemed that full review and approval was not necessary. Funding required for survey construction was provided by the Department of Paediatrics at Washington University School of Medicine.

The content of the survey is available in the appendix. Sites self-reported as urban, suburban, or rural - these terms were not defined. Sites also self-identified with NACHRI designation listed as follows: children's general hospital, children's specialty hospital, children's unit in a general hospital, or not identified by NACHRI. For the rest of the manuscript, we will use the term " free standing children's hospitals," which will include both children's general hospitals and children's specialty hospitals." The vast majority of centres in this study are provided blood products from a centralized blood services in the U.S., where approximately $85 \%$ of RBCs are leukocyte reduced. In the U.S., according to the American Association of Blood Bank, plasma can be thawed and stored at 2-6C for up to 5 days.

\section{$\underline{\text { Results }}$}

Of the 62 surveys sent to children's hospitals, we received completed surveys from 50 unique sites. If more than one response was received from a single site, we accepted the first fully completed survey. Four of these sites did not have an MTP policy and their survey 
responses were not included in this analysis; therefore, we analysed 46 survey responses. Please see Table 1 for site demographics. The specialty role of the 46 survey respondents were as follows: $28 \%(13 / 46)$ were intensive care unit directors or attending physicians (consultant), 26\% (12/46) were trauma directors or trauma program managers, $20 \%(9 / 46)$ were transfusion medicine directors, $11 \%(5 / 46)$ were emergency department directors or attending physician, $9 \%(4 / 46)$ were other, and $7 \%$ (3/46) were administrative coordinators.

\section{MTP activation criteria}

Most sites, $89 \%$ (41/46), listed physician discretion as a criterion for activating their MTP. The survey offered several laboratory data as options for activation criteria, but only a small minority, 3 of the 46 respondents $(7 \%)$, listed specific laboratory threshold as an activation criterion: haemoglobin 4\% (2/46), international normalized ratio (INR) 4\% (2/46), base deficit 4\% (2/46), prothrombin time (PT) 2\% (1/46), partial thromboplastin time (PTT) 2\% (1/46), platelet count $2 \%(1 / 46)$, rapid/standard thromboelastography (TEG) values $0 \%$. In contrast, more sites, $17 \%(8 / 46)$, answered "yes" for specific vital signs or physical exam findings as MTP activation criteria. The specific vital signs or physical exam findings answers were as follows: systolic blood pressure 15\% (7/46), heart rate 11\% (5/46), mean arterial pressure $9 \%(4 / 46)$, respiratory rate $7 \%(3 / 46)$, Glasgow Coma Scale $7 \%(3 / 46)$, oxygen saturation $4 \%(2 / 46)$, tissue oxygen saturation $2 \%(1 / 46)$, and temperature $0 \%$. When asked if mechanism of injury was considered an activation criterion according to their MTP, $9 \%(4 / 46)$ responded "yes," with 9\% (4/46) specifying penetrating injury and 4\% (2/46) specifying blunt injury. Interestingly, 26\% (12/46) responded with "other" MTP activation criteria; seven sites included a threshold amount of transfused blood product as activation criteria (Table 2).

\section{Blood products administered according to MTP}




\section{General Questions}

When asked, "Does your MTP policy specify a ratio of plasma to Red Blood Cell (RBC)," 89\% (41/46) replied "yes." Sixty one percent (28/46) of sites indicated cryoprecipitate was included in their MTP policy. Whole blood was available at $9 \%(4 / 46)$ of sites. There was a maximum RBC storage age at $15 \%(7 / 46)$ sites. Specific stipulations for maximum storage days were as follows: three of the 46 sites specified 7 days, one site specified 8 days, two sites specified 14 days, and one site specified a maximum of 21 days.

Plasma:RBC ratios

Table 3 displays the plasma:RBC ratios suggested for transfusion in the first and subsequent blood packages according to hospital MTP. In the first blood package, when defining "high" plasma:RBC ratio as $\geq 1: 2,78 \%(36 / 46)$ specified high ratios and $4 \%$ specified $(2 / 46)$ low ratios; $13 \%(6 / 46)$ of sites did not specify a ratio or had missing data; and $4 \%(2 / 46)$ replied with "other." Two of the sites that reported a policy to transfuse a "high" plasma:RBC ratio also reported that plasma:RBC ratios varied according to the patient's weight. One site used a 1:1 plasma:RBC ratio for children $<30 \mathrm{~kg}$ but used a $0.5: 1$ ratio for children $>30 \mathrm{~kg}$. The other site specified a 1:1 plasma:RBC ratio for patients less than 5 kilograms or greater than $50 \mathrm{~kg}$ but used a 1:2 ratio for patients that were 5 to $49 \mathrm{~kg}$. For subsequent blood packages, $85 \%$ (39/46) stipulated high ratios and $2 \%$ (1/46) stipulated low ratios. For these subsequent blood packages, respondents indicated "not applicable" or "did not specify ratio" in $11 \%$ $(5 / 46)$ and "other" in $2 \%(1 / 46)$ of responses. One of the sites that reported a policy to transfuse a "high" plasma:RBC ratio also responded that their policy for subsequent blood packages varied ratios based on weight. 
Of the respondents with answers that could be grouped as "high plasma:RBC" or "low plasma:RBC" ratios, 93\% (27/29) of free standing children's hospitals indicated that their MTP's specify a high plasma:RBC ratio in the first group of blood units, while $100 \%$ (9/9) of general hospitals with children's units specify a high ratio.

\section{Platelet to $R B C$ ratios}

Table 4 displays the platelet to RBC ratios reported for the first and subsequent blood packages. Of the 46 respondents with MTPs, 65\% (30/46) reported that a ratio of apheresis platelets to RBC units was specified in the first group of blood units. The survey provided answers in terms of both apheresis platelets and donor platelets, specifying one apheresis platelet unit was equal in volume and platelet number to approximately 5 random donor platelet units. When defining "high" ratio as $\geq 1: 10$ apheresis platelet to $\mathrm{RBC}$ or $\geq 1: 2$ random donor platelet (RDP) to RBC, 54\% (25/46) had high ratios (with one site specifying varying ratios based on weight), 35\% (16/46) did not specify a ratio, $11 \%(5 / 46)$ classified as "other," and none were identified as low ratio. Of the sites classified as "other," two sites had varying ratios based on weights and three sites did not specify any platelets. For subsequent blood packages, $54 \%(25 / 46)$ had high ratios, 37\% (17/46) had missing data or not applicable, $7 \%$ (3/46) replied "other," and none had low ratios. Of the sites that replied "other," 2 sites had varying ratios based on weight and one did not include platelets in the second blood package.

\section{Intravenous haemostatic agents}

Among respondents, haemostatic agents incorporated into MTPs were as follows: $24 \%$ (11/46) recombinant factor VII (rFVII), 15\% (7/46) antifibrinolytics (tranexamic acid and/or aminocaproic acid), 13\% (6/46) fibrinogen concentrates, 11\% (5/46) prothrombin complex 
concentrates, and 24\% (11/46) did not include haemostatic agents in their MTP. Of the 46 respondents, $15 \%(5 / 34)$ of freestanding children's hospitals use antifibrinolytics in their MTPs versus $17 \%(2 / 12)$ of general hospitals with children's unit.

\section{Laboratory data after MTP activation}

Fifty percent (23/46) of centres require laboratory measures after MTP activation; however, one site answered "yes" but then answered "no" to subsequent questions. Specified laboratory measures were as follows: INR/PT/PTT 49\% (22/45), haemoglobin or haematocrit $47 \%$ (21/45), fibrinogen $44 \%$ (20/45), platelet count $40 \%$ (18/45), ionized calcium $29 \%$ (13/45), potassium 24\% (11/45), base deficit 18\% (8/45), lactate 18\% (8/45), TEG 9\% (4/45), and none require thromboelastometry (ROTEM). Respondents were asked if their site's MTP specified the frequency of post activation laboratory data and 26\% (12/46) centres responded "yes." The laboratory data frequency was based on the amount of product given at 6 centres $(13 \%)$ while the frequency of labs was time-based at $6(13 \%)$ of 46 centres. Laboratory data were to be obtained every 30 minutes at 11\% (5/46) and every 6 hours at $2 \%(1 / 46)$ of centres.

\section{Blood products immediately available}

Type O RBC units were stored in an immediately available location at $89 \%(41 / 46)$ of sites. The location of immediate release type O RBC storage was as follows: emergency department $37 \%$ (17/46), operating room $20 \%$ (9/46), intensive care unit $11 \%(5 / 46)$, blood bank $63 \%(29 / 46)$, and $7 \%$ (3/46) stated "other" (hall fridge $=1$, neonatal intensive care unit $=1$, and missing data $=1$ ).

Thawed plasma units were stored in an immediately available location at $48 \%(22 / 46)$ of centres: blood bank 46\% (21/46), emergency department 4\% (2/46), and $2 \%(1 / 46)$ of 
centres stated "other" (specified as satellite blood bank). Specifically, 54\% (18/33) of free standing children's hospital had thawed plasma immediately available while $33 \%(4 / 12)$ of general hospitals with children's units had thawed plasma immediately available.

\section{MTP activation frequency}

Respondents were asked how often the MTP was activated from January 1, 2013 through December 31, 2013. Of the 46 respondents, 27 (59\%) reported an exact number of activations, however there were missing data from 4 centres. The median number of activations was 6, with IQR (3-10). In contrast, 41\% (19/46) of respondents reported estimated activations, although 4 respondents answered "unknown." The median number of estimated MTP activations was 5, with IQR (4-5).

\section{Compliance measures}

The following committees were responsible for audit of MTP activations: transfusion committee 41\% (19/46), trauma committee 30\% (14/46), "other" 9\% (blood bank $=2$, emergency transfusion subcommittee $=1$, multidisciplinary team $=1$ ), no committee $2 \%$ (1/46), and $17 \%(8 / 46)$ did not know. Compliance to specific metrics was tracked at $54 \%$ (25/46) of sites. These specific metrics were not surveyed.

\section{Discussion}

This survey is the first of our knowledge to report on massive transfusion protocol policies among children's hospitals in the United States and Canada. As expected, the responses revealed a wide range of clinical practice.

Results from our survey indicate that MTP activation criteria are most often based on "physician discretion," a non-specific entity. Activation criteria such as vital signs, laboratory 
values, and previous amount of blood products transfused were uncommon. A possible explanation for this finding may be the lack of data in children that identify paediatric patients at risk for massive bleeding or the need for massive transfusion. Seven sites included a specific amount of transfused red blood cells to be administered as one mechanism for MTP activation. There was significant variation between these seven sites, as some use only RBCs, while other sites use all blood products transfused as MTP activation criteria. Neff et al recently published that more than $40 \mathrm{ml} / \mathrm{kg}$ of all blood products (total volume) administered indicated an increased risk of mortality (Neff, Cannon et al., 2015), potentially indicating that total blood product volume administered may be a more appropriate definition of massive transfusion. This approach allows for the blood bank to identify a patient who may have massive bleeding when the clinical team has potentially not recognized this or has inadvertently forgotten to activate an MTP. Additional investigation focusing on predictors of massive bleeding in children or the need for massive transfusion is necessary to improve outcomes. Accurate prediction of who is at risk for massive bleeding may allow for more rapid treatment and even prevention of the need for massive transfusion, which may have the potential to improve outcomes.

We were quite interested in the ratio of plasma, red blood cells, and platelets that were specified in the MTPs. A major principle of DCR includes early empiric balanced transfusion (1:1:1 unit ratio of red blood cells (RBCs), plasma, and platelets) termed "Haemostatic Resuscitation" (Spinella and Holcomb, 2009). A recent prospective randomized clinical trial of severely injured adults who were predicted to require massive transfusion revealed that more patients in the 1:1:1 ratio of plasma:platelets:RBC group compared with the 1:1:2 ratio of plasma, platelets and RBCs group achieved haemostasis and fewer experienced death due to exsanguination. However, there was not a significant difference in mortality at 24 
hours or at 30 days between the two groups (Holcomb, Tilley et al., 2015). Although the adult literature is stronger, there are a few small studies that have evaluated higher plasma:RBC ratios in children. One retrospective study by Nosanov indicated that there was not an association between higher plasma:RBC ratios and survival in a cohort of children transfused greater than $50 \%$ of their blood volume (Nosanov, Inaba et al., 2013). A second prospective pilot study in children with burn injuries compared a $1: 1$ to $1: 4$ plasma:RBC ratio strategies, with more plasma and less RBCs administered in the 1:1 group, but no difference in PT/PTT, INR, haemoglobin, or platelets between groups (Palmieri, Greenhalgh et al., 2013).

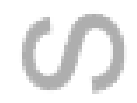

Our results show a large majority of sites specified a high $(\geq 1: 2)$ plasma or platelet to RBC ratio and incorporate cryoprecipitate in their MTP. Sites frequently targeted 1:1 ratios for both plasma and platelet to $\mathrm{RBC}$ ratios. We believe this practice reflects the application of adult evidence to children. It is possible that many aspects of DCR can and should be applied to children; however, high quality studies are needed in children to determine if these adult guidelines are appropriate for the resuscitation of paediatric patients with massive bleeding.

There was no statistical difference between the percentage of free standing children's hospitals and general hospitals with children's units MTPs regarding high plasma:RBC ratios. We expected the opposite, with higher ratios being used for children at general adult hospitals that have paediatric units within them, since there are predominantly adult data that supports this approach and the increased frequency of MTP activation at adult hospitals allowing for the immediate availability of plasma. Interestingly, two sites called for varying plasma:RBC ratio based on weight. The rationale for this is not evident, but may be based 
on the concept that hemostasis changes with development and transfusion requirements may be different based on age.

In regard to hemostatic adjunct use, only 15 percent of sites used antifibrinolytics (tranexamic acid or aminocaproic acid) in their MTPs. The CRASH-2 trial, a randomized controlled study enrolling 20,000 adults, revealed a $32 \%$ reduction in death due to bleeding when tranexamic acid was given within one hour of injury, with no increase in vascular occlusive events (collaborators, Shakur et al., 2010). Although antifibrinolytics reduce mortality in the adult trauma population, the use of antifibrinolytics has not been well studied in children. While the use and safety of antifibrinolytics has been shown in children requiring cardiac surgery, craniofacial surgery, and scoliosis surgery (Schouten, van de Pol et al., 2009), no mortality analyses were performed. A recent retrospective study of paediatric trauma patients cared for in a combat hospital revealed an association between tranexamic acid use and improved survival, although the data set were limited as there was not information on blood loss, laboratory response to tranexamic acid, or tranexamic dose timing (Eckert, Wertin et al., 2014). Although $15 \%$ of sites use antifibrinolytics, $23.9 \%$ (11/46) of sites in this survey used recombinant factor VIla (rFVIla). A recent systematic review of transfusion interventions in critical bleeding requiring massive transfusion found that rFVIIa had no benefit in mortality outcomes in critical bleeding or trauma, and that a modest reduction in RBC transfusion and blood loss was off set by the increased risk of thromboembolic events (McQuilten, Crighton et al., 2015). Clinical trials are needed to determine the indications and appropriate dosing of antifibrinolytics and other hemostatic agents for children with massive bleeding. 
Fifty percent (23/46) of sites required laboratory measures after MTP activation and, as expected, nearly all of those sites required INR/PT/PTT, as these labs are traditionally used to monitor coagulopathy. In contrast, only four sites required the use of TEG. Research is required to determine which laboratory measures are most accurate at measuring coagulopathy and are effective at permitting a goal directed therapy strategy for the treatment of haemorrhagic shock in children.

While $89 \%$ of sites had type O RBC units stored in an immediately available location (most commonly in the blood bank or emergency department), only $48 \%$ of sites had thawed plasma units stored in an immediately available location. Our recent survey of 132 US adult tertiary care centres indicated that $66 \%$ have thawed plasma immediately available (Camazine, Hemmila et al., 2015). We expected general hospitals to be more likely to have thawed plasma available, as there may be difficulty providing pre-thawed plasma at free standing children's hospitals due to lower frequency of MTP events compared to large adult facilities, and the increased waste of product that may result. However, there was no statistical difference between the percentage of free standing children's hospital with thawed plasma immediately available compared to general hospitals with children's units $(p=$ 0.314). This may be due to our modest sample size.

The low use of whole blood available was expected, as there has been a transition from the use of whole blood to component therapy over the last several decades. Component therapy is the primary transfusion approach due to concerns for resource utilization and safety (Spinella and Holcomb, 2009). However, a retrospective study from adult military data demonstrated that warm fresh whole blood is independently associated with improved survival in trauma patients compared to component therapy (Spinella, Perkins et al., 2009). 
A randomized controlled trial (RTC) in children with congenital heart disease indicated that when whole blood was transfused postoperatively, there was a reduction in bleeding compared to those transfused blood components (Manno, Hedberg et al., 1991). On the other hand, the complications associated with whole-blood transfusion, such as renal failure or respiratory distress has been thought to be attributed to the white blood cells in the wholeblood units. Therefore, the use of leukocyte-reduced whole blood with platelet sparing filtration may be safer, as is routinely done at the Children's Hospital of Philadelphia for Cardiac surgery (Murdock, Berseus et al., 2014; Jobes, Sesok-Pizzini et al., 2015).

There was a maximum RBC storage age at $15 \%$ (7/46) sites. Recent randomized controlled trials have indicated that the use of fresher RBCs does not improve outcomes (Fergusson, Hebert et al., 2012; Lacroix, Hebert et al., 2015; Steiner, Ness et al., 2015). However, none of these trials been performed in patients with traumatic injury nor those who require a significant amount of blood products for resuscitation, where the risk of the storage lesion may be highest (Steiner and Stowell, 2009). Additional studies in the paediatric population are needed to determine if whole blood or fresh RBCs improve outcomes in children with severe bleeding.

This study had some limitations. Due to some non-mandatory questions, there were missing data. In addition, we allowed estimates, as well as the answer "unknown," in regard to MTP activations, which did not allow for accurate estimations of MTP frequency. The decision to allow non-mandatory questions and estimates was made in an effort to increase survey response, although we recognize this may introduce bias. Many of the sites that responded seemed to be from larger paediatric institutions, as $89 \%$ had ECMO capabilities and $91 \%$ performed solid organ transplants, which limits the generalizability to sites that do not offer 
these services. Only $20 \%$ of survey respondents were transfusion medicine directors; however, the survey was initially sent to paediatric trauma directors who could delegate completion of the survey to another person in a different specialty at the site if that individual was more familiar with specific aspects of their MTP (i.e. the transfusion medicine directors). The wide range of survey respondents may be evidence of broad interest in MTPs among multiple specialties. Due to length of survey limitations, we were not able to expand on certain topics, such as the timing and indications for rFVIla or TXA use, or if laboratory data were used to alter plasma to RBC ratios. Finally, this study surveyed policy rather than actual practice, which may be different, as other studies have demonstrated that physicians may perceive adherence to recommendations or guidelines to be better than it actually is (Brunkhorst, Engel et al., 2008; Oderda, Shiozawa et al., 2014).

Implementation and support of MTPs is resource intensive, further highlighting the importance of identifying which transfusion strategies are most effective. This is especially relevant at children's hospitals that have a low frequency of massive bleeding, where the cost of making blood products available for the application of DCR may exceed its utility. Further study is needed to examine the benefits versus possible detriments of MTPs, as there is also evidence that adults who receive a higher plasma to $\mathrm{RBC}$ ratio and who are at low risk for life-threatening haemorrhage are at increased risk for organ failure and increased number of ventilator days compared to similar patients transfused a low plasma to RBC ratio (Borgman, Spinella et al., 2011). This also highlights the importance of recognizing which patients are at low risk for life-threatening haemorrhage, which has not yet been characterized for the paediatric population. 
A prospective observational study is currently being performed that will collect epidemiologic, practice range, and outcomes for all children who have an MTP activated. These data from over 300 children will serve as preliminary data for trials in children that can assess the efficacy and safety of DCR principles in children. Costs of multiple trials can be reduced by conducting well designed comparative effectiveness trials (Spinella, Leonard et al., 2015).

\section{Conclusion}

In conclusion, there is a wide variation in MTPs among paediatric hospitals with regard to both activation criteria and transfusion support. Most units have embraced the principles of DCR but have not formalized when to initiate it and whether the same protocols should be used in trauma and non-trauma. This underscores the need for future prospective studies assessing the epidemiology of MTP activations, which therapies are most commonly used in children, and associated outcomes according to the cause of massive bleeding to determine the most effective resuscitation methods for paediatric populations in order to improve outcomes and therapeutic safety for massive bleeding.

Acknowledgement: We sincerely appreciate Dr. Heidi Doughty's insightful review of our manuscript.

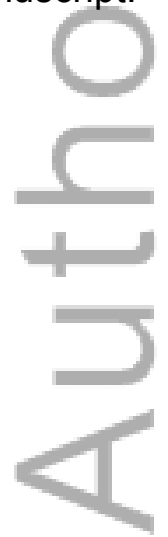




\section{Bibliography}

Agrawal, A., A.B. Beethe, et al. (2013). Continuous hemoglobin monitoring during massive blood transfusion in a multivisceral pediatric transplant patient. J Clin Anesth 25, 578581.

Andrew, M., P. Vegh, et al. (1992). Maturation of the hemostatic system during childhood. Blood 80, 1998-2005.

Appel, I.M., B. Grimminck, et al. (2012). Age dependency of coagulation parameters during childhood and puberty. J Thromb Haemost 10, 2254-2263.

Borgman, M.A., P.C. Spinella, et al. (2011). The effect of FFP:RBC ratio on morbidity and mortality in trauma patients based on transfusion prediction score. Vox Sang 101, 4454.

Borgman, M.A., P.C. Spinella, et al. (2007). The ratio of blood products transfused affects mortality in patients receiving massive transfusions at a combat support hospital. $J$ Trauma 63, 805-813.

Brunkhorst, F.M., C. Engel, et al. (2008). Practice and perception--a nationwide survey of therapy habits in sepsis. Crit Care Med 36, 2719-2725.

Camazine, M.N., M.R. Hemmila, et al. (2015). Massive transfusion policies at trauma centers participating in the American College of Surgeons Trauma Quality Improvement Program. J Trauma Acute Care Surg 78, S48-53.

Center for Disease Control, C. (2010). WISQARS Leading Causes of Death Reports, 1999 2007. http://webappa.cdc.gov/sasweb/ncipc/leadcaus10.html.

Chidester, S.J., N. Williams, et al. (2012). A pediatric massive transfusion protocol. J Trauma Acute Care Surg 73, 1273-1277.

collaborators, C.-t., H. Shakur, et al. (2010). Effects of tranexamic acid on death, vascular occlusive events, and blood transfusion in trauma patients with significant haemorrhage (CRASH-2): a randomised, placebo-controlled trial. Lancet 376, 23-32.

Cotton, B.A., B.K. Au, et al. (2009). Predefined massive transfusion protocols are associated with a reduction in organ failure and postinjury complications. J Trauma 66, 41-48; discussion 48-49.

Cotton, B.A., O.L. Gunter, et al. (2008). Damage control hematology: the impact of a trauma exsanguination protocol on survival and blood product utilization. J Trauma 64, 11771182; discussion 1182-1173.

Demetriades, D., J. Murray, et al. (2004). Trauma fatalities: time and location of hospital deaths. J Am Coll Surg 198, 20-26.

Dente, C.J., B.H. Shaz, et al. (2009). Improvements in early mortality and coagulopathy are sustained better in patients with blunt trauma after institution of a massive transfusion protocol in a civilian level I trauma center. J Trauma 66, 1616-1624.

Dressler, A.M., C.M. Finck, et al. (2010). Use of a massive transfusion protocol with hemostatic resuscitation for severe intraoperative bleeding in a child. J Pediatr Surg 45, 1530-1533.

Eckert, M.J., T.M. Wertin, et al. (2014). Tranexamic acid administration to pediatric trauma patients in a combat setting: The pediatric trauma and tranexamic acid study (PEDTRAX). J Trauma Acute Care Surg 77, 852-858.

Fergusson, D.A., P. Hebert, et al. (2012). Effect of fresh red blood cell transfusions on clinical outcomes in premature, very low-birth-weight infants: the ARIPI randomized trial. JAMA 308, 1443-1451. 
Gunter, O.L., Jr., B.K. Au, et al. (2008). Optimizing outcomes in damage control resuscitation: identifying blood product ratios associated with improved survival. $J$ Trauma 65, 527-534.

Guzzetta, N.A. and B.E. Miller (2011). Principles of hemostasis in children: models and maturation. Paediatr Anaesth 21, 3-9.

Hendrickson, J.E., B.H. Shaz, et al. (2012). Implementation of a pediatric trauma massive transfusion protocol: one institution's experience. Transfusion 52, 1228-1236.

Holcomb, J.B., B.C. Tilley, et al. (2015). Transfusion of plasma, platelets, and red blood cells in a 1:1:1 vs a 1:1:2 ratio and mortality in patients with severe trauma: the PROPPR randomized clinical trial. JAMA 313, 471-482.

Holcomb, J.B., C.E. Wade, et al. (2011). Increased Plasma and Platelet to Red Blood Cell Ratios Improves Outcome in 466 Massively Transfused Civilian Trauma Patients (vol $=248$, pg 447, 2008). Annals of Surgery 253, 392-392.

Jaffray, J. and G. Young (2013). Developmental hemostasis: clinical implications from the fetus to the adolescent. Pediatr Clin North Am 60, 1407-1417.

Jobes, D.R., D. Sesok-Pizzini, et al. (2015). Reduced transfusion requirement with use of fresh whole blood in pediatric cardiac surgical procedures. Ann Thorac Surg 99, $1706-1711$.

Lacroix, J., P.C. Hebert, et al. (2015). Age of transfused blood in critically ill adults. N Engl J Med 372, 1410-1418.

Manno, C.S., K.W. Hedberg, et al. (1991). Comparison of the hemostatic effects of fresh whole blood, stored whole blood, and components after open heart surgery in children. Blood 77, 930-936.

McQuilten, Z.K., G. Crighton, et al. (2015). Transfusion interventions in critical bleeding requiring massive transfusion: a systematic review. Transfus Med Rev 29, 127-137.

Murdock, A.D., O. Berseus, et al. (2014). Whole blood: the future of traumatic hemorrhagic shock resuscitation. Shock 41 Suppl 1, 62-69.

Neff, L.P., J.W. Cannon, et al. (2015). Clearly defining pediatric massive transfusion: cutting through the fog and friction with combat data. J Trauma Acute Care Surg 78, 22-28; discussion 28-29.

Nosanov, L., K. Inaba, et al. (2013). The impact of blood product ratios in massively transfused pediatric trauma patients. Am J Surg 206, 655-660.

Nylund, C.M., M.A. Borgman, et al. (2009). Thromboelastography to direct the administration of recombinant activated factor VII in a child with traumatic injury requiring massive transfusion. Pediatr Crit Care Med 10, e22-26.

O'Keeffe, T., M. Refaai, et al. (2008). A massive transfusion protocol to decrease blood component use and costs. Arch Surg 143, 686-690; discussion 690-681.

Oderda, G.M., A. Shiozawa, et al. (2014). Physician adherence to ACR gout treatment guidelines: perception versus practice. Postgrad Med 126, 257-267.

Palmieri, T.L., D.G. Greenhalgh, et al. (2013). Prospective comparison of packed red blood - cell-to-fresh frozen plasma transfusion ratio of 4: 1 versus 1: 1 during acute massive burn excision. J Trauma Acute Care Surg 74, 76-83.

Perkins, J.G., A.P. Cap, et al. (2009). An evaluation of the impact of apheresis platelets used in the setting of massively transfused trauma patients. J Trauma 66, S77-84; discussion S84-75.

Schouten, E.S., A.C. van de Pol, et al. (2009). The effect of aprotinin, tranexamic acid, and aminocaproic acid on blood loss and use of blood products in major pediatric surgery: a meta-analysis. Pediatr Crit Care Med 10, 182-190. 
Spinella, P.C. and J.B. Holcomb (2009). Resuscitation and transfusion principles for traumatic hemorrhagic shock. Blood Rev 23, 231-240.

Spinella, P.C., J. Leonard, et al. (2015). Massive Transfusion Epidemiology \& Outcomes in Children Study: pediatrics.wustl.edu/matic.

Spinella, P.C., J.G. Perkins, et al. (2009). Warm fresh whole blood is independently associated with improved survival for patients with combat-related traumatic injuries. J Trauma 66, S69-76.

Steiner, M.E., P.M. Ness, et al. (2015). Effects of red-cell storage duration on patients undergoing cardiac surgery. N Engl J Med 372, 1419-1429.

Steiner, M.E. and C. Stowell (2009). Does red blood cell storage affect clinical outcome? When in doubt, do the experiment. Transfusion 49, 1286-1290.

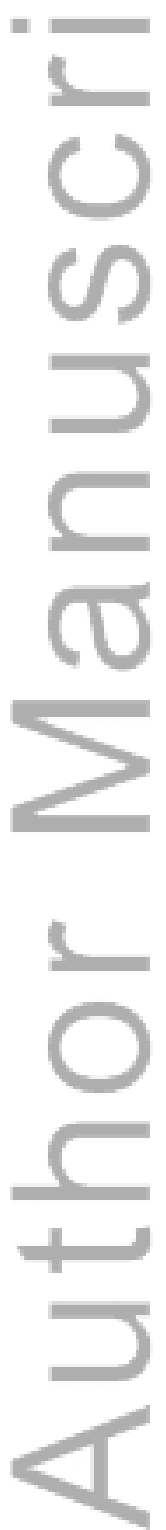


Table 1. Institution Demographics

$N=46$

\begin{tabular}{|c|c|c|}
\hline & \# & $\%$ \\
\hline \multicolumn{3}{|l|}{ NACHRI Designation } \\
\hline Freestanding Children's Hospital $^{*}$ & 34 & $74 \%$ \\
\hline Children's General Hospital & 16 & $35 \%$ \\
\hline Children's Speciality Hospital & 18 & $39 \%$ \\
\hline Children's Units in a General Hospital & 12 & $26 \%$ \\
\hline \multicolumn{3}{|l|}{ Location } \\
\hline Urba & 36 & $78 \%$ \\
\hline Suburban & 7 & $15 \%$ \\
\hline Rural & 3 & $7 \%$ \\
\hline \multicolumn{3}{|l|}{ Capabilities } \\
\hline $\mathrm{ECMO}$ & 41 & $89 \%$ \\
\hline Solid organ transplant & 42 & $91 \%$ \\
\hline 1 & & \\
\hline \multicolumn{3}{|l|}{ Trauma Designation } \\
\hline ACS level one & 30 & $65 \%$ \\
\hline State verified level one & 22 & $48 \%$ \\
\hline
\end{tabular}

*"Freestanding Children's Hospital" is not a specific NACHRI designation, but is used throughout the manuscript to include both children's general hospitals and children's specialty hospitals.

KEY: NACHRI = National Association of Children's Hospitals and Related Institutions. $\mathrm{ECMO}=$ extracorporeal membrane oxygenation. $\mathrm{ACS}=$ American College of Surgeons 


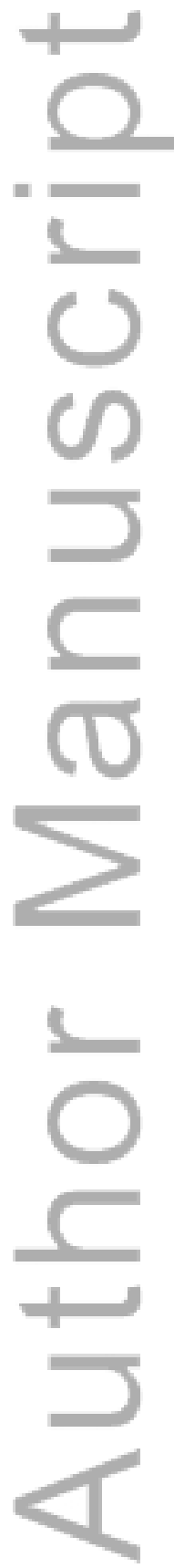


Table 2. The amount of transfused blood product to be given before MTP activation at seven different sites

Amount transfused equals more than $50 \mathrm{ml} / \mathrm{kg}$

Thirty $\mathrm{ml} / \mathrm{kg}$ and on-going uncontrolled bleeding

Administration of $>40 \mathrm{ml} / \mathrm{kg}$ PRBC (2 sites)

RBC transfusion requirement of greater than $20 \mathrm{ml} / \mathrm{kg}$ in the first hour of resuscitation or approaching $40 \mathrm{ml} / \mathrm{kg}$ in 12 hours or $80 \mathrm{ml} / \mathrm{kg}$ in 24 hours

Actual or anticipated administration of $>40 \mathrm{ml} / \mathrm{kg}$ PRBC in 2 hours or less.

Administration of one estimated blood volume (approximately 10 units of RBC in 24 hours in an adult, $80 \mathrm{ml} / \mathrm{kg}$ for paediatric patients) or $>50 \%$ of total blood volume in 2 hours and continuing need for transfusion. 
Table 3. Plasma:RBC ratios in first and subsequent blood packages*

\begin{tabular}{|l|c|c|c|c|c|c|}
\hline & $\begin{array}{c}1: 1 \\
\text { plasma:RBC }\end{array}$ & $\begin{array}{c}\mathbf{2 : 3} \\
\text { plasma:RBC }\end{array}$ & $\begin{array}{c}\mathbf{1 : 2} \\
\text { plasma:RBC }\end{array}$ & $\begin{array}{c}\mathbf{1 : 3} \\
\text { plasma:RBC }\end{array}$ & $\begin{array}{c}\text { Ratio varies } \\
\text { with weight }\end{array}$ & $\begin{array}{c}\text { No plasma in } \\
\text { first package }\end{array}$ \\
\hline $\begin{array}{l}\mathbf{1}^{\text {st }} \text { blood } \\
\text { package } \\
\text { Plasma:RBC }\end{array}$ & $54.3 \%(25 / 46)$ & $4.3 \%(2 / 46)$ & $15.2 \%(7 / 46)$ & $4.3 \%(2 / 46)$ & $4.3 \%(2 / 46)$ & $4.3 \%(2 / 46)$ \\
\hline $\begin{array}{l}\text { Subsequent } \\
\text { blood package } \\
\text { Plasma:RBC }\end{array}$ & $65 \%(30 / 46)$ & $2.2 \%(1 / 46)$ & $15.2 \%(7 / 46)$ & $2.2 \%(1 / 46)$ & $2.2 \%(1 / 46)$ & $\begin{array}{c}\text { None } \\
\text { specified }\end{array}$ \\
\hline
\end{tabular}

*Does not include sites with missing data or whose MTP does not specify a ratio.

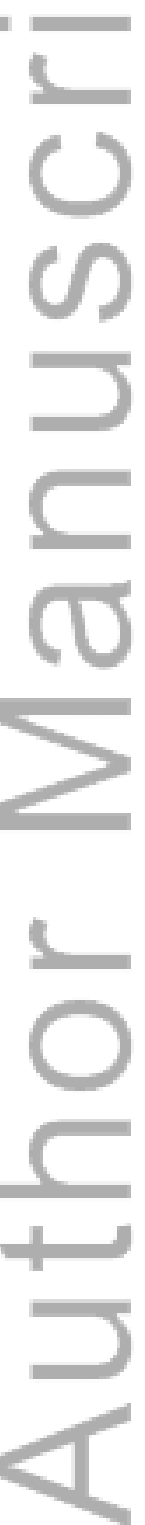


Table 4. Platelet:RBC ratios in first and subsequent blood packages.

\begin{tabular}{|r|c|c|c|c|c|c|c|}
\hline & $\begin{array}{c}1: 5 \\
\text { apheresis } \\
\text { platelet:RBC } \\
\text { apheresis } \\
\text { platelet: } \\
\text { RBC or 1:1 } \\
\text { RDP:RBC }\end{array}$ & $\begin{array}{c}1: 6 \\
\text { apheresis } \\
\text { platelet:RBC }\end{array}$ & $\begin{array}{c}1: 8 \\
\text { apheresis } \\
\text { platelet:RBC }\end{array}$ & $\begin{array}{c}1: 10 \\
\text { apheresis } \\
\text { platelet: } \\
\text { RBC or 1:2 } \\
\text { RDP:RBC }\end{array}$ & $\begin{array}{c}\text { Ratio varies } \\
\text { with weight }\end{array}$ & $\begin{array}{c}\text { No platelets } \\
\text { in first } \\
\text { package }\end{array}$ \\
\hline $\begin{array}{r}\text { 1st } \\
\text { Platelet:RBC }\end{array}$ & $4.3 \%(2 / 46)$ & $\begin{array}{c}34.8 \% \\
(16 / 46)\end{array}$ & $2.2 \%(1 / 46)$ & $4.3 \%(2 / 46)$ & $6.5 \%(3 / 46)$ & $6.5 \%(3 / 46)$ & $6.5 \%(3 / 46)$ \\
\hline $\begin{array}{r}\text { Subsequent } \\
\text { blood } \\
\text { package }\end{array}$ & $4.3 \%(2 / 46)$ & $\begin{array}{c}32.6 \% \\
(15 / 46)\end{array}$ & $2.2 \%(1 / 46)$ & $4.3 \%(2 / 46)$ & $10.9 \%(5 / 46)$ & $4.3 \%(2 / 46)$ & $2.2 \%(1 / 46)$ \\
\hline
\end{tabular}

Key: RBC=Red Blood Cell, RDP=Random Donor Platelet

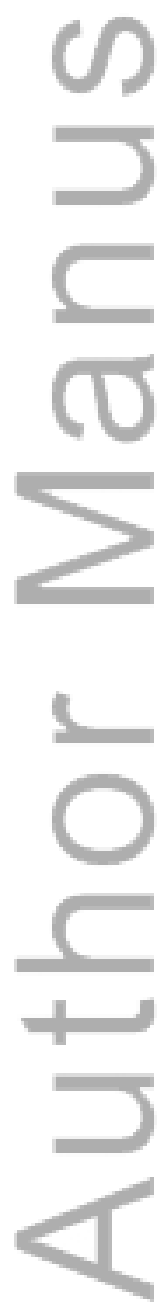




\section{University Library}

\section{- M M N E R VA A gateway to Melbourne's research publications}

Minerva Access is the Institutional Repository of The University of Melbourne

Author/s:

Horst, J;Leonard, JC;Vogel, A;Jacobs, R;Spinella, PC

Title:

A survey of US and Canadian hospitals' paediatric massive transfusion protocol policies

Date:

2016-02-01

Citation:

Horst, J., Leonard, J. C., Vogel, A., Jacobs, R. \& Spinella, P. C. (2016). A survey of US and Canadian hospitals' paediatric massive transfusion protocol policies. TRANSFUSION MEDICINE, 26 (1), pp.49-56. https://doi.org/10.1111/tme.12277.

Persistent Link:

http://hdl.handle.net/11343/290889 